\title{
Orígenes del conectivismo como nuevo paradigma del aprendizaje en la era digital
}

\section{Origins of connectivism as a new learning paradigm in the digital era}

\begin{abstract}
Roberto Sánchez-Cabrero ID
Universidad Alfonso X el Sabio, Madrid, España

Óscar Costa-Román

Universidad Isabel I la católica, Madrid, España

Lidia Mañoso-Pacheco

Universidad Antonio de Nebrija, Madria, España

Miguel Novillo-López

Universidad Nacional de Educación a Distancia, Madrid, España

Francisco Pericacho-Gómez

Universidad Antonio de Nebrija, Madrid, España

\section{Resumen}

Objetivo: Mostrar las raíces teóricas del conectivismo en las principales teorías históricas sobre la adquisición de conocimiento y el aprendizaje, ya que se trata del paradigma educativo más útil para entender el aprendizaje en la era digital y los procesos de e-learning. Método: Se realiza una exhaustiva recopilación de fuentes originales pertinentes, seleccionadas en base a su relevancia y significado dentro de cada corriente teórica. A través de esta revisión se presenta al conectivismo como una evolución respecto a las escuelas anteriores y no una revolución teórica en el ámbito pedagógico. Se valora al Psicoanálisis y la Escuela de la Gestalt como antecedentes más remotos y se considera la influencia del Instruccionismo, Constructivismo, Teoría del Caos, Neurociencia, Teoría de redes y Sistemas Adaptativos Complejos como referentes directos. Se continúa con el análisis de las influencias recibidas por las distintas escuelas teóricas coetáneas y actuales, como la Teoría de la Conversación, la Teoría del Actor-Red, la Red de aprendizaje (Network Learning), e-learning 2.0., Microlearning, Nano-learning, Universidad 2.0., Currículum 2.0., Pedagogía 2.0., y el Navegacionismo, para completar los fundamentos teóricos sobre los que se apoya el Conectivismo. Conclusión y discusión: Se confirman las conexiones teóricas del conectivismo con las teorías expuestas y se pronostica cada vez mayor relevancia en el ámbito educativo de este paradigma por su adecuación a la realidad social actual.
\end{abstract}

Palabras clave: Conectivismo, Paradigmas educativos, Redes sociales, Nuevas tecnologías, elearning.

\begin{abstract}
Objective: To review the main theories about the main theories about knowledge and learning, as the theoretical origins of Connectivism, as the most useful educational paradigm to understand learning in the digital era and e-learning processes. Method: Through an exhaustive compilation of pertinent original sources, selected based on their relevance and meaning within each theoretical stream, Connectivism is presented as an evolution with respect to previous schools and not a theoretical revolution in the pedagogical field. Psychoanalysis and the School of Gestalt, on their part, are regarded in this research as the earliest antecedents, and the influence of Instructionism, Constructivism, Chaos Theory, Neuroscience, Network Theory and Theory of Complex Adaptive Systems is also introduced in the present paper, as these theories are considered direct predecessors. It continues with the analysis of the influences received by the different contemporary and contemporary theoretical schools, namely the Theory of Conversation, the Actor-network theory, Network Learning, elearning 2.0., Microlearning, Nano-learning, University 2.0., Curriculum 2.0., Pedagogy 2.0. and Navigationism. These schools complete the theoretical foundations on which Connectivism is based. Conclusion and discussion: The theoretical connections of Connectivism with the exposed theories are confirmed, and it is predicted an increasing relevance in the educational field of this paradigm due to its adaptation to the current social reality.
\end{abstract}

Keywords: Connectivism, Educational Paradigms, Social Networking, New technologies, elearning.

Open Access:

ISSN: 0124-2121

E-ISSN: $2665-2420$

Editor:

Patricia Martínez Barrios Universidad Simón Bolivar

Correspondencia:

Roberto Sánchez

robsan9@gmail.com

Recibido: 2018-10-18

Aceptado: 20-11-18

Publicado: 02-01-19

DOI:

http://dx10.17081/edu hum.21.36.3265

Copyright () 2019 Educación Y Humanismo 


\section{Introducción}

El aprendizaje en la era digital se caracteriza por una permanente conexión del aprendiz al entorno. Este entorno, no solo se basa en la cercanía física o teórica con el aprendiz, sino también en su posibilidad de acceso mediante distintas redes virtuales, como internet o las redes sociales digitales (Twitter, Facebook, etc.) (Nobles, Londoño, Martínez, Ramos, Santa \& Cotes, 2016; Maldonado, 2017).

Un efecto colateral de esta permanente conexión ha sido la ruptura de la barrera de la distancia en la educación. La educación mediante la conexión a entornos virtuales ya no puede denominarse 'educación a distancia', sino que ha evolucionado a una modalidad cualitativamente diferente denominada aprendizaje electrónico o e-learning (Aprendizaje electrónico: el que se realiza a distancia a través de canales electrónicos e internet principalmente). Las principales diferencias entre la educación a distancia tradicional y el e-learning, según Rivera, Alonso \& Sancho (2017), serían:

- El e-learning solo es posible si hay una fuerte presencia de las TIC (tecnologías de información y comunicación) y especialmente de Internet

- Se crean comunidades educativas formadas entre profesores y alumnos, solo alumnos, o solo profesores

- Los alumnos son autónomos.

- Se basa en la educación asincrónica

- No tiene por qué haber distancia física entre el profesor y sus alumnos

Los modelos clásicos y actuales sobre el aprendizaje fracasan al tratar de explicar este aprendizaje, puesto que gran parte del mismo no se encuentra dirigido a un fin concreto, ni depende directamente de la voluntad y voluntariedad del aprendiz, sino que surge directamente de la propia fusión del aprendiz con su entorno y la adopción de sus principios, modos de vida, actitudes, etc. (Fuentes, 2017).

El conectivismo es el único enfoque teórico actual que es capaz de ofrecer una comprensión adecuada de este tipo de aprendizaje; el resto de paradigmas teóricos tienen un alcance limitado para mostrar la influencia del mundo digital interconectado sobre la persona que aprende, puesto que para ellos el aprendizaje siempre es individual y voluntario, cualidades contrarias al aprendizaje actual en la era digital (Islas \& Delgadillo, 2016).

Haciendo un trabajo de reflexión sobre las aportaciones de los principales teóricos del Conectivismo es posible encontrar una base teórica sobre la que asentar unos principios y un enfoque para comprenderlo. Hablar de enfoque y no de teoría sobre la adquisición de conocimiento o del aprendizaje no es un acto trivial, ya que al hacerlo esquivamos la mayoría de las críticas actuales al Conectivismo; incluidas las aportadas por Downes, uno 
de los fundadores y principales promotores de este enfoque teórico de la educación (Downes, 2008; Forster, 2008; Zapata-Ros, 2015).

Esta perspectiva de abordaje conectivista supone hacer énfasis sobre la utilidad pragmática de las afirmaciones conectivistas. El Conectivismo sirve para interpretar y comprender los procesos asociados al aprendizaje y la adquisición de conocimiento en el mundo actual, especialmente en lo referido a la evolución tecnológica de las redes sociales y a ambientes multiformales de aprendizaje, mediación y recreación de situaciones de aprendizaje ligados a las tecnologías a través del e-learning. No se debe, por tanto, utilizar los principios conectivistas para explicar todas las formas de aprendizaje y adquisición de conocimiento, ya que ello supone encontrar lagunas en sus principios que son cubiertas por teorías anteriores, a las que el Conectivismo complementa a modo de adecuación al mundo tecnológico digital (Downes, 2016; Flórez, Pérez \& Amaya, 2017).

El Conectivismo, en su intento para comprender cómo se adquiere conocimiento y cómo se produce el aprendizaje, define la mente humana como una red que se adapta al entorno. Por lo tanto, el aprendizaje sería el proceso de formación de redes a través de conexiones entre distintos nodos, y el conocimiento residiría en dichas redes. El papel del aprendiz sería activo y creativo, ya que tiene la necesidad de actualizarse continuamente a su entorno cambiante a través de realizar nuevas conexiones, reconocer patrones y aprender a través de la experiencia en la toma de decisiones (Siemens 2006, Siemens \& Conole, 2011).

Un concepto capital en el Conectivismo es la idea del conocimiento como voluble, inestable, incontrolable y en continua expansión, lo que supone que escape del control total de la persona, pudiendo residir en sus redes externas (comunidades, dispositivos digitales, etc.) y estar en permanente cambio. El Conectivismo es, por lo tanto, no solo una concepción de conocimiento y aprendizaje humano individual, sino también un enfoque para entender la mentalidad colectiva de una red de personas, una comunidad o una sociedad en base a un mismo principio, la generación de ecologías de redes en constante cambio y desarrollo (Aguilar \& Mosquera, 2015).

Es fácilmente reconocible la similitud de la concepción de la mente humana y el conocimiento según el Conectivismo con la red de redes (Internet) y con el surgimiento y rápida expansión de las redes sociales digitales como Twitter, Facebook, etc., lo cual no deja de ser lógico, ya que se trata de creaciones humanas, que basarían su éxito en la gran adecuación que presentan para la satisfacción de la necesidad humana de creación y utilización de redes sociales para adaptarse al medio y, por lo tanto, con un gran beneficio secundario de su uso destinado al desarrollo social, lo que incluye el aprendizaje social no formal. Ahí reside la principal importancia del Conectivismo, ya que es, sin lugar a dudas, el enfoque teórico psicopedagógico más robusto y en mejores condiciones para describir y explicar el mundo actual, en el que los entornos sociales y digitales se mezclan, dando lugar a nuevas formas de adquisición de conocimiento y aprendizaje, para las cuales otras teorías anteriores resultan insuficientes o incompletas; empezando por las nuevas características de la adquisición de conocimiento en esta era digital, que lo hacen voluble, 
desordenado, horizontal, democrático, pero sobre todo, en constante y exponencial expansión.

El propósito de esta revisión teórica es mostrar cómo el conectivismo no ha surgido de forma independiente del resto de escuelas teóricas, sino que es el fruto de todas ellas al actualizarse a la era digital. El conectivismo parte, por lo tanto, de una serie de antecedentes teóricos que nacen en la psicología y en la pedagogía y que tienen su continuidad en el conectivismo, como se va a mostrar a lo largo de esta revisión.

\section{Metodología}

Esta revisión de los orígenes del conectivismo en teorías sobre el aprendizaje y la adquisición de conocimiento anteriores, parte de la necesidad de algunos miembros de la comunidad científica actual en el ámbito pedagógico y de historia de la educación de reflejar un recorrido histórico plausible que muestre la evolución de este ámbito hasta el día de hoy, y justificar al conectivismo como enfoque teórico actual por cuestiones pragmáticas.

Diversos expertos en educación, pedagogía, psicología, historia y nuevas tecnologías aplicadas a la educación se han planteado reflejar las raíces teóricas de los postulados del conectivismo, por lo que se inició la búsqueda exhaustiva de fuentes originales pertinentes a través de las distintas bases de datos científicas disponibles en la red (Google Scholar, Psycnet, Eric, entre otros) y las mejores y más reconocidas revistas científicas de los distintos ámbitos teóricos según Web of Science y Scopus. El criterio aplicado de selección de documentos para el análisis y las referencias seleccionadas se estableció en la relevancia internacional de cada trabajo y autor dentro de cada paradigma y escuela teórica, y la citación de dichos trabajos como claves, influyentes o paradigmáticos para la comprensión de las bases teóricas de cada corriente teórica.

Finalmente, basándose en la relevancia histórica de las distintas referencias encontradas, se elabora esta revisión, fruto del intenso y dedicado trabajo de distintos autores con diversos perfiles y experiencias profesionales.

Desarrollo y discusión: Principales referencias teóricas del conectivismo

\subsection{Los antecedentes más remotos}

El más remoto de los antecedentes del Conectivismo se podría localizar en la práctica psicoterapéutica del Psicoanálisis (Freud, 1900, citado en Freud, 1984). El Conectivismo comparte algunas curiosas similitudes con esta controvertida escuela psicológica, lo que 
justifica poder hacer un ligero paralelismo entre ambas. Tanto el Psicoanálisis como el Conectivismo comparten las siguientes características destacadas:

- Ambas escuelas nacen de la creación principalmente de un solo autor: Freud (1900, citado en Freud, 1984) y Siemens (2004), aunque posteriormente recibieron enriquecedoras aportaciones posteriores de distintos autores.

- Ambas escuelas teóricas, tal y como sucede con el surgimiento de nuevas corrientes teóricas en la mayoría de ciencias, surgen con la intención de llegar ahí donde no logran explicar las teorías científicas contemporáneas el comportamiento humano. La principal diferencia estriba en que Freud, principalmente busca comprender las motivaciones humanas llegando a la idea del inconsciente. Mientras que Siemens, por su parte, busca completar las lagunas e inadecuaciones que dejan las teorías clásicas del aprendizaje, sobre todo en relación a un rápido y amplio incremento de conocimiento, lo que Siemens considera como su principal talón de Aquiles (Siemens \& Conole, 2011).

- Ambas escuelas han recibido feroces críticas como teorías científicas, siendo más recomendable su aplicación como perspectivas; terapéutica en el caso del Psicoanálisis y pedagógica en el caso del Conectivismo.

- Ambas escuelas tuvieron una rápida propagación y generaron numerosas influencias, tanto en su campo como en otros, por lo que focalizaron un gran interés científico en su época.

- Finalmente, la principal similitud entre ambas se establece en la gran importancia dada al funcionamiento mental humano en forma de red. Por un lado, en el Psicoanálisis es clave como método terapéutico la Asociación Libre de Ideas (Freud y Breuer, 1895, citado en Schutt, 1995). Por otro lado, el Conectivismo define el aprendizaje como el proceso de conectar nodos o fuentes de información. Por lo tanto, podemos considerar que ambas escuelas consideran un funcionamiento muy similar de la mente humana a la hora de razonar o aprender en forma de red y de generación de conexiones entre ideas.

Otro de los antecedentes más remotos del Conectivismo puede ser encontrado en la Escuela de la Gestalt. Los teóricos de esta escuela consideran el aprendizaje como un proceso de desarrollo de nuevas ideas o como una modificación de las antiguas (Wertheimer \& Riezler, 1944). Para ello el aprendiz debe tener un papel activo en su aprendizaje, ya que llega al aprendizaje intencionalmente, explorando su entorno y circunstancias, siendo creativo. Esta idea enlaza con la idea del Conectivismo de la necesidad de "reforma y ajuste" de la red de aprendizaje. Siemens considera que el aprendiz forma redes de conocimiento que adapta a sus necesidades actuales y que posteriormente modifica al son de sus propias circunstancias cambiantes (Siemens \& Conole, 2011).

Al igual que en la Escuela de la Gestalt, en el Conectivismo también se considera una visión global de la realidad con un fin puramente pragmático. En ambas escuelas teóricas 
los conocimientos están supeditados a la visión global que generan, por lo que son menos importantes que las visiones en las que se funden y su significado es mutable según las circunstancias. El aprendiz forma su propia realidad usando los elementos (conocimientos) de los que dispone; la actitud activa y creativa del aprendiz, por lo tanto, es clave a la hora de generar aprendizaje, que además será totalmente personal y único respecto a cualquier otro aprendiz.

Por otra parte, conviene resaltar que algunas de las principales leyes de la Escuela de la Gestalt (Similitud, proximidad, impregnancia, etc.) son leyes que reflejan la forma particular que tiene la mente humana de realizar conexiones y asociaciones (Rock \& Palmer, 1990). Leyes que también son usadas por el Conectivismo para reflejar cómo se forma y modifica una red de conocimiento en cada momento y para cada aprendiz (Siemens \& Conole, 2011).

\subsection{Los antecedentes en las ciencias pedagógica y psicológica}

El continuo desarrollo del conocimiento en las ciencias psicológica y pedagógica ha supuesto una gran fuente de referencias para el Conectivismo. Numerosos autores de gran relevancia han servido de inspiración reconocida para las aportaciones de algunos de los más importantes autores conectivistas (Siemens, Downes, etc.). Autores como Bruner, Ausubel, Piaget, Bandura, Gagné, etc. y corrientes científicas como la Teoría de Redes, la Neurociencia, el Instruccionismo, la Teoría del Caos, etc. han puesto algunas de las primeras piedras para la elaboración de los principales axiomas conectivistas.

Ante la tradicional yuxtaposición de Instruccionismo frente al Constructivismo en la educación, el Conectivismo se posiciona, sin duda alguna, mucho más cercano al Constructivismo (Duffy \& Jonassen, 2013). No obstante, eso no implica que alguna de las ideas del Instruccionismo no hayan servido de referentes. Considerando el Instruccionismo, tal y como lo define Papert (1995), como la "expresión de la creencia de que el camino hacia un aprendizaje mejor pasa por el perfeccionismo de la instrucción (p. 151)" se puede observar cómo el Conectivismo coincide con esta corriente teórica en que es la práctica y el perfeccionamiento la que lleva a un conocimiento más profundo y complejo al aprendiz, aunque para el Conectivismo este proceso no consiste en la perfección de la instrucción, sino en la mejora en la toma de decisiones del aprendiz basada en la mejora de su red de conocimiento (Rodríguez, 2016). Sería la riqueza de la red la que llevaría a una visión más compleja y a una profundización más centrada en los intereses del aprendiz.

Por lo que respecta al Constructivismo, esta corriente es identificable con la tercera metáfora del aprendizaje según Mayer (Mayer, 1992, citado por Zapata-Ros, 2015) como puede observarse en la Tabla 1: 
Tabla 1.

Las tres metáforas del aprendizaje

\begin{tabular}{llll}
\hline Aprendizaje como Enseñanza & Foco instruccional & Resultados \\
\hline $\begin{array}{l}\text { Adquisición de } \\
\text { respuestas }\end{array}$ & $\begin{array}{l}\text { Suministro de } \\
\text { Feedback }\end{array}$ & $\begin{array}{l}\text { Centrado en el currículo } \\
\text { (Conductas correctas) }\end{array}$ & $\begin{array}{l}\text { Cuantitativos } \\
\text { (Fuerza de las } \\
\text { asociaciones) }\end{array}$ \\
\hline $\begin{array}{l}\text { Adquisición de } \\
\text { conocimiento }\end{array}$ & $\begin{array}{l}\text { Transmisión de } \\
\text { información }\end{array}$ & $\begin{array}{l}\text { Centrado en el currículo } \\
\text { (Información apropiada) }\end{array}$ & $\begin{array}{l}\text { Cuantitativos } \\
\text { (Cantidad de } \\
\text { información) }\end{array}$ \\
\hline $\begin{array}{l}\text { Construcción de } \\
\text { significado }\end{array}$ & $\begin{array}{l}\text { Orientación del } \\
\text { procesamiento } \\
\text { cognitivo }\end{array}$ & $\begin{array}{l}\text { Centrado en el estudiante } \\
\text { (Procesamiento significativo) }\end{array}$ & $\begin{array}{l}\text { Cualitativos } \\
\text { (Estructura del } \\
\text { conocimiento) }\end{array}$ \\
\hline
\end{tabular}

Fuente: Mayer, 1992, citado en Zapata-Ros, (2015).

Dentro de la perspectiva constructivista de la construcción de significados en el aprendizaje conviene diferenciar diversidad de enfoques teóricos de diversos autores que también han servido de puntos de apoyo para las teorías conectivistas.

El primer enfoque teórico constructivista que funciona de antecedente del Conectivismo es la Escuela de la Gestalt, con Von Wertheimer y Köhler a la cabeza, de la que ya se habló en los antecedentes remotos en este mismo artículo.

El principal impulsor del Constructivismo en la educación, Piaget, introduce la importancia de la interacción con el medio social en su desarrollo de la Teoría Constructivista del Aprendizaje. Según Piaget (1954) el aprendizaje se va construyendo en base a la maduración y la experiencia del aprendiz con su entorno social y físico, y gracias a ello se amplía y modifican las estructuras mentales en otras más complejas.

Gagné (1971) es otro de los autores que sirve de inspiración al Conectivismo, al considerar que existen condicionantes internos y externos que regulan el proceso de aprendizaje, por lo que comparte la idea conectivista de ecología y de adaptación al medio social. Los condicionantes internos funcionan como almacén para la adquisición de capacidades que son requisitos previos para el aprendizaje; los condicionantes externos hacen referencia al contexto que facilita el aprendizaje.

Otro de los autores más relevantes en el Constructivismo, Bruner (1966), introduce el concepto de Aprendizaje por Descubrimiento, lo que supone aceptar la idea del aprendizaje no intencional y la aparición de retos al aprendiz que pueden modificar sus intereses y su forma de resolver los problemas. Siemens (2004) desarrolla esta idea como la definición completa de un problema a través de la combinación de los distintos puntos de vista de los agentes implicados mediante su creatividad individual, que acaban descubriendo una visión más profunda del problema a partir de la combinación casual de sus intereses particulares. Por otra parte, Bruner (2001) considera como un aspecto clave 
en el aprendizaje el diálogo activo alumno-profesor, lo que supone la creación de una red de aprendizaje externa generadora de conocimiento, tal y como afirman las ideas conectivistas.

Vygotsky también ha supuesto una fuerte inspiración conectivista. Vygotsky (1932) desarrolla la idea de competencia y considera los factores sociales como desencadenantes externos del aprendizaje a modo de red externa de conocimiento; aunque su inspiración más clara seguramente sea la idea de la Zona de Desarrollo Próximo, idea que define como:

La distancia entre el nivel de desarrollo real del niño tal y como puede ser determinado a partir de la resolución independiente de problemas y el nivel más elevado de desarrollo potencial tal y como es determinado por la resolución de problemas bajo la guía del adulto o en colaboración con sus iguales más capacitados (Vygotsky, 1932, citado en Wertsch, 1988; p. 84).

Esta idea de Vygotsky (1932) de la Zona de Desarrollo Próximo enlaza directamente con el funcionamiento de una red conectivista de conocimiento y coincide con el principio conectivista de "la capacidad de aumentar el conocimiento es más importante que lo que ya se sabe" (Siemens, 2006; p. 31).

Finalmente, Ausubel (1964) concibe el aprendizaje como un proceso de atribución de significado. Lo nuevo debe enlazar con lo anterior y, de esta forma, lo transforma y lo convierte en un conocimiento más complejo y profundo. El aprendiz también gana en el proceso capacidad de adaptación a nuevas situaciones porque tiene un conocimiento más sólido, donde los conocimientos más elementales sostienen a los más complejos en una estructura jerárquica (Andamiaje) similar al funcionamiento conectivista de red.

La metáfora del aprendizaje como Construcción de Significado propia del Constructivismo ha supuesto la evolución natural del Cognitivismo hacia un currículo centrado en el alumno (Abrio \& Bermudez, 2017). Desde esta perspectiva el alumno tiene un papel activo y protagonista sobre su propio aprendizaje, ya que es él mismo el que construye y estructura su propio aprendizaje a través de la construcción de significado a los conocimientos que va adquiriendo. La perspectiva constructivista del cognitivismo ha sido la predominante desde los años 80 , aproximadamente, hasta la actualidad en el ámbito científico educativo, y representa multitud de cambios en la configuración educativa del aprendizaje en los colegios en la actualidad, sirviendo de referencia a nuevas metodologías educativas que se están implantando en la actualidad como la Educación por proyectos, los rincones de trabajo, etc. (Cathalifaud, 2014).

El Conectivismo comparte gran parte de esta visión global del aprendiz en el Constructivismo, ya que también le otorga el papel protagonista de su aprendizaje y le dota de un papel activo en la elección de los contenidos y la forma de estructurarlos con un significado único y propio. No obstante, difieren en un aspecto sustancial, y es que el Conectivismo rechaza la idea de la constante construcción de significados. Según afirma 
Siemens (2006) 'ho siempre construimos (lo que supone una carga cognitiva), pero sí estamos constantemente conectando (p. 27)". Por lo tanto, según los postulados conectivistas, el aprendiz no siempre está construyendo, lo que supone un intento de organización del caos del conocimiento asequible para el aprendiz, sino que, en ocasiones, simplemente se enriquece navegando entre ese caos sin la necesidad de organizarlo, 0 incluso desorganizando lo organizado, es decir deconstruyendo lo construido significativamente. Por ejemplo, es fácil comprender esta navegación entre el caos sin la construcción constante de significados en el aprendiz si se piensa en cualquier persona navegando sin rumbo definido de enlace a enlace a través de Internet, simplemente guiado por su interés momentáneo y curiosidad.

Esta crítica del Conectivismo al Constructivismo nos introduce a otro de los antecedentes psicopedagógicos del Conectivismo, que es la Teoría del Caos aplicada a la Educación. Según la Teoría del Caos, lo social está inacabado y se acoge a fenómenos distorsionadores y caóticos que desde el orden generan desorden y viceversa (Castells, 1999), por lo que la educación, como ciencia social, debería fundamentarse en tal perspectiva, ya que la idea del orden, como portador de la verdad científica, es una idea caduca, ya superada, y no aplicable a las ciencias sociales, que siempre están en constante construcción y deconstrucción. Tal y como afirma Colom (2005):

La epistemología propia de las ciencias humanas y sociales y, por tanto, el discurso acerca de la educación, tras la modernidad, debe conjugar complejidad y desorden, es decir, debe prescindir ya de la simplicidad (la paranoia analítica de la modernidad), y del orden como portador de certezas, que siguen siendo cualidades propias de la ciencia del S. XIX ( $p$. 1327).

Desde el punto de vista cognitivo, se considera a la creatividad como el motor caótico de la inteligencia (Colom, 2005), que abre las puertas al desorden que genera el conocimiento adquirido en el aprendiz y lo lleva a nuevos y complejos logros de aprendizaje que pueden volver a ordenarse en un nivel superior, y así indefinidamente.

Es fácil ver la inspiración del Conectivismo en estos principios de la Teoría del Caos. El Conectivismo, al igual que la Teoría del Caos, no considera que todo el aprendizaje está dirigido, genera orden cognitivo y es intencional. Por otro lado, también coinciden en la fluctuación constante del conocimiento que hace que lo actual sea perecedero y termine siendo el germen de lo nuevo. No obstante, el alcance de la Teoría del Caos para definir la adquisición de aprendizaje del aprendiz es más limitada, ya que no considera los puntos de apoyo que suponen los nodos de conexiones a la hora de dar definición al aprendizaje de cada aprendiz, por lo que no habría ninguna base sobre la que se sostenga la toma de decisiones (Arenas, 2018). Según la Teoría del Caos no habría una estructura clara que guie al aprendiz y, por lo tanto, todos podrían ser iguales o igualmente diferentes. En el Conectivismo esa red de conocimiento formada por nodos es la que estructura el aprendizaje y el conocimiento, y sirve de base de forma no determinista para futuros aprendizajes con una personalidad fluctuante, pero concreta y personal, que no estaría determinada desde la Teoría del Caos. 
Los avances científicos actuales en la Neurociencia también han supuesto un fuerte apoyo para las teorías conectivistas. Los descubrimientos en neurociencia cognitiva en relación al funcionamiento de las neuronas espejo descubiertas por Rizzolatti, Fadiga, Matelli, Bettinardi, Paulesu, Perani y Fazio. (1996) confirman la idea conectivista de que el conocimiento se puede materializar en la mente humana sin la mediación de los aspectos simbólicos, y por lo tanto sin la creación constante de significados.

Inicialmente se pensó en las neuronas espejo como ejecutoras de propiedades de imitación en el ser humano, pero se ha descubierto que van mucho más allá. Según numerosos estudios este sistema neuronal, presente sobre todo en áreas frontal parietal de la corteza cerebral, permite al ser humano sentir como propias sensaciones, emociones e incluso acciones que son percibidas en los demás. Estos hallazgos sugieren que múltiples sistemas en los humanos pueden estar dotados de mecanismos neuronales de reflejo, tanto para la integración y la diferenciación de los aspectos perceptivos y motrices de las acciones realizadas por uno mismo, como de los demás (Mukamel, Ekstrom, Kaplan, Iacoboni \& Fried, 2010). Las conexiones con el Conectivismo son claras, se refuerza la idea de que el conocimiento está distribuido, no sólo en un ser humano, sino a través de nuestra empatía intra-especie, que también puede residir en dispositivos no humanos (una película, una canción, un buscador de internet, etc.), y se refuerza la idea que el ser humano está continuamente conectando, más allá de su análisis cognitivo, expandiendo su conocimiento en forma de red.

La principal referencia para asociar el Conectivismo con las ciencias sociales o sociopsicológicas se puede localizar en la Teoría de redes. Esta teoría tiene su origen en la Escuela de la Gestalt y recibió su primer impulso con los trabajos de psicología de grupos de Lewin (1938) y los estudios sociométricos de Moreno (1962). Los teóricos de esta propuesta pretenden analizar el funcionamiento grupal de una red de personas partiendo del supuesto de que lo que la gente siente, piensa y hace como grupo tiene su origen y se manifiesta en las pautas de relaciones situacionales que se dan entre ellos y no en sus atributos individuales (Lozares, 2005). Por lo tanto, una red tendría una entidad propia y diferente que sus miembros, se puede analizar y no sería reducible a la aportación de cada individuo del grupo.

Esta idea de red social implica un conocimiento común, lo que Siemens (2011) define como 'Sabiduría de las multitudes', y un funcionamiento en red de un grupo, al igual que ocurre en el Conectivismo. Tiene numerosas aplicaciones en otras ciencias como la economía, con los análisis micro y macro económicos; con las matemáticas, a través de la Teoría de Grafos (Konig, 1936); o incluso con la informática y las redes sociales electrónicas.

La última de las referencias a considerar de gran relevancia para el Conectivismo podemos establecerla en los Sistemas Adaptativos Complejos (Complex Adaptive System: CAS). Los Sistemas Adaptativos Complejos son un campo de estudio interdisciplinar que abarca diversas ciencias como la psicología, sociología, economía, genética, biología, inteligencia artificial, etc. y son comúnmente usados para describir grupos de elementos 
interrelacionados que reaccionan de forma adaptativa a los cambios ambientales que les afectan. El término fue adoptado inicialmente por el Instituto de Santa Fe en la década de los 90 con Holland, Gell-Man y Forrest a la cabeza, pero se popularizó rápidamente por su capacidad para explicar la adaptación ambiental de sistemas complejos de diversas ciencias (Holland, 1996). Según Levin (2002) las principales propiedades de los Sistemas Adaptativos Complejos son la diversidad e individualidad de los componentes, las interacciones locales y específicas entre esos componentes y un proceso autónomo que utiliza los resultados de esas interacciones para replicar o mejorar un subconjunto de esos componentes; aunque la propiedad que realmente define un sistema adaptativo complejo es, sin duda, la adaptación. Que el sistema se adapte significa que aprende, que existe una selección competitiva y que solo sobreviven los más adecuados al ambiente actual convirtiéndose en norma para la replicación (Forrest \& Jones, 1994).

La idea de aprendizaje de los Sistemas Adaptativos Complejos es fundamentalmente la misma del Conectivismo a la hora de considerar el funcionamiento de una red o ecología, por lo que toma prestados muchas de sus afirmaciones. El Conectivismo también considera que la red de conocimiento se adapta al ambiente y que se seleccionan la toma de decisiones más aptas para el ambiente actual dando forma a un darwinismo de redes o sistemas.

También el concepto no definido de conocimiento según el Conectivismo tiene las cualidades de un sistema adaptativo complejo. Es decir, no tiene forma concreta, ya que depende del ambiente que se encuentre; está en continuo cambio o adaptación y se expande y replica constantemente.

\subsection{Referencias actuales en teorías comunicativas y la llegada de la revolución digital}

El paso final en esta revisión teórica debe darse en la actualidad. En aquellos movimientos teóricos más actuales, coetáneos con el Conectivismo, y que de alguna manera han dejado su impronta sobre él; ya sea inspirando sus teorías o modificando y perfeccionando las bases teóricas sobre las que se sustenta. Muchos de estos enfoques teóricos parten de ideas similares y tienen como función describir la forma de aprender de la nueva sociedad altamente influenciada por las nuevas tecnologías de la información y el conocimiento, aunque su alcance, por uno u otro motivo, ha sido notablemente inferior al Conectivismo, que ha tenido como un gran factor a su favor una política de publicaciones abiertas, que ha favorecido una mayor repercusión internacional y en el ámbito científico (Santamaría, 2010).

La primera de las referencias a considerar puede ser hallada en la Teoría de la Conversación, originalmente formulada por Pask (1975) y posteriormente reestructurada dialógicamente por Laurillard (1993, 1999, 2002). Según esta teoría el diálogo entre profesor y aprendiz permite la construcción e intercambio de conocimientos entre ambos interlocutores, de tal forma que el aprendiz reconstruye el significado de un determinado concepto expuesto por el profesor, y partiendo de ese punto, el profesor debe valorar el 
aprendizaje producido utilizando para ello sus propios métodos de evaluación. Por lo tanto, se podría considerar que existe una realimentación intrínseca en el proceso de aprendizaje a través del diálogo, ya que ha de estar presente al menos una fase de adaptación del sistema de aprendizaje por parte del aprendiz (Martín, García \& Ramírez, 2004). Este proceso conversacional serviría para hacer explícito el conocimiento y promover la reflexión a modo de aprendizaje socrático. Tanto el Conectivismo como la Teoría de la Conversación se fundamentan en el establecimiento de relaciones como fuente de conocimientos, ya sea el hecho de relacionar ideas como el de relacionarse con otras personas y reflexionar sobre ello (Lima, 2017).

Otra aportación de relevancia sería la Teoría del Actor-Red (Actor- Network Theory, ANT), también conocida como Ontología del Actante-Rizoma. Se trata de un enfoque sociológico surgido en los años 80, pero desarrollada teóricamente a finales de los 90 con los trabajos de Latour (1999) y Law (1999) principalmente. La Teoría del Actor-Red considera a las máquinas, a los objetos, e incluso a los discursos, como actantes, al mismo nivel que los seres humanos en un sistema simétrico en el que cobra una gran relevancia los aspectos tecnológicos (Vidal, 2016). Esta teoría supone que todos estamos conectados sin distinguir entre humanos y no-humanos, por lo que la tecnología, los procesos sociales y los seres humanos son asociados y estudiados al mismo nivel en los análisis sociales.

La llegada de Internet y la revolución digital supone el surgimiento de nuevas teorías basadas en su funcionamiento y las relaciones que se generan a través de los medios digitales. Ésta es la idea idea de Red de aprendizaje (N-learning: Network Learning) de Polsani (2003). Inspirado en los trabajos de Harasim (1995). Polsani considera que el surgimiento de Internet ha modificado nuestra forma de aprender, que ha derivado en la aparición de un lugar de producción de conocimiento global virtual a la que nos conectamos, enriquecemos, y de la cual aprendemos a lo largo de toda la vida. Esta red de conocimiento y aprendizaje estaría estructurada en distintas capas de conocimientos a las que accederíamos según nuestras necesidades. Tanto el Conectivismo como las teorías de Polsani comparten la idea de que el conocimiento y el aprendizaje están distribuidos, pueden residir en dispositivos no humanos y son considerados como entidades vivas y diferentes a la suma de sus elementos.

La aparición de la Web 2.0, y su notable influencia sobre las nuevas metodologías pedagógicas, ha derivado en la aparición de nuevos enfoques teóricos que han influenciado algunas concepciones e ideas conectivistas.

Downes (2005), el principal impulsor del Conectivismo junto con Siemens, desarrolla la idea del e-learning 2.0., al que atribuye muchas de las cualidades que también desarrolla para el Conectivismo, lo que supone un cambio sustancial respecto al e-learning tradicional. Según Downes (2005) El e-learning 2.0 supone la integración de la Web 2.0 sobre la enseñanza, lo que significa que el aprendiz participa de su propio aprendizaje, es capaz de crear contenidos que comparte con otros, se puede aprender a través de otros canales más informales y los conocimientos son alterables; no se organizan de manera jerárquica y son modificables y adaptables a las necesidades del aprendiz. En definitiva, el 
elearning 2.0 supone una participación activa del aprendiz electrónico sobre su propio aprendizaje, que además le convierte en parte del aprendizaje de otros aprendices con los que se conecta (Osuna \& Almenara, 2015).

Dos nuevas modalidades de la educación derivadas del e-learning, que tienen en común el estar basadas en pequeñas porciones de aprendizajes, también suponen una referencia clara del Conectivismo. Se hace referencia al Microlearning y al Nano-learning.

Por lo que respecta al Microlearning o Microaprendizaje, según Hug (2007) es posible programar aprendizajes muy efectivos con pequeñas actividades con objetivos a muy corto plazo. Estas actividades se basan en microcontenidos que son posteriormente utilizados en conocimientos más amplios o a largo plazo (Salinas \& Marín, 2015). El Conectivismo también contempla la existencia y utilidad de estos microaprendizajes, sobre todo en el aprendizaje no intencional o creativo y suponen en muchos casos los primeros pasos para desarrollos más avanzados en nuevas materias o en temáticas diferentes, funcionando a modo de exploradores de rutas nuevas para la red de conocimiento.

En cuanto al nano-learning o n-learning (No confundir con N-learning: Network Learning), supone una miniaturización del aprendizaje incluso mayor que el Microlearning. Haciendo una analogía con la nanotecnología se atomiza el aprendizaje a su mínima expresión (dedicación continuada de incluso poco más de un minuto) lo que garantiza un gran rendimiento en atención y una comprensión por descubrimiento muy alta, permitiendo una gran diversificación y personalización del aprendizaje por parte del aprendiz (Masie, 2006; Imran \& Kowalski, 2014).

Siguiendo con las nuevas tendencias basadas en las nuevas tecnologías, Barnes y Tynan (2007) plantean la necesidad de una nueva universidad, puesto que los actuales alumnos universitarios ya son usuarios de la Web 2.0. y les parece insuficiente una formación centrada en el profesor y su conocimiento. El término Web 2.0 surgió para referirse a las nuevas páginas de Internet que se diferenciaban de los sitios web más tradicionales englobados bajo la denominación Web 1.0. La característica diferencial es la participación colaborativa de los usuarios. Esta nueva universidad la denominan Universidad 2.0 y consiste en hacer énfasis en las nuevas tecnologías de red social en el ámbito universitario (Esteve, 2016; Ganino, 2017). De esta forma el alumno podrá aportar al conocimiento a la vez que se enriquece de él y se evita un aislamiento de los contenidos universitarios de la realidad del alumno. En esta nueva universidad, al igual que en el Conectivismo, se considera que tendrá gran relevancia el conocimiento informal, que tradicionalmente es visto en el ámbito universitario como un aprendizaje menor.

Asociado a estas ideas de adaptar todo el ámbito educativo a la nueva realidad virtual 2.0., otros autores se atreven a hablar de la existencia de un nuevo Currículum 2.0., con las características de estas nuevas tecnologías (Sobrino, 2014). El Currículo se negocia entre alumnos y profesor y estaría guiado por las necesidades del aprendiz (Bawden, Robinson, Anderson, Bates, Rutkauskiene, \& Vilar, 2008). Gracias a este currículo 
personalizado los alumnos desarrollarían mayores habilidades de gestión y acceso al conocimiento al estar adaptado a su ritmo y necesidades, lo que supondría una aplicación de los principios conectivistas.

Según autores como McLoughlin y Lee (2007), las nuevas tecnologías de la información aplicadas al conocimiento y la enseñanza demandan cambios sustanciales a la hora de hacer la pedagogía en el mundo actual. Esta nueva manera de afrontar la enseñanza es denominada Pedagogía 2.0 y debe ser acorde con las necesidades del aprendiz en el mundo actual (Hardman, 2015). Algunas de las más importantes demandas actuales son la participación en comunidades y redes de aprendizaje (ya sea sociales o virtuales), la personalización de tareas de aprendizaje para el ritmo diferente de cada aprendiz y el énfasis en la producción de conocimiento, es decir, aportar al conocimiento a la vez que se enriquece de él (McLoughlin \& Lee, 2008). Estas características de aprendizaje personalizado, ruptura del aislamiento del contexto de enseñanza, y el conocimiento horizontal y democrático son también ideas capitales en el Conectivismo.

Introducir nuevas tecnologías dentro de los contextos pedagógicos puede suponer una importante aportación a la pedagogía, más allá de la tradicional conceptualización como distractores. Brown (2006) considera el uso de estos dispositivos de comunicación (móviles, tabletas, etc.) como una actividad relacionada directamente con el manejo eficiente de la información y la comunicación asequibles. El aprendiz deberá relacionarse con otros aprendices e instructores, asociar conocimientos desde su propia perspectiva con su conocimiento previo, compartir los conocimientos descubiertos y colaborar con otros aprendices el proceso de su propio aprendizaje. Brown (2006) denomina a esta nueva perspectiva como Navegacionismo, donde el manejo de información y la interacción social continuada son sus pilares (Organista, McAnally \& Lavigne, 2013).

\subsection{El conectivismo en el momento actual}

Tal y como se ha apuntado anteriormente, el conectivismo como tal es una corriente muy joven que se ha desarrollado en plena era digital. Solórzano y García (2016), indican, al reflexionar sobre las afirmaciones de Sangrà y Wheleer (2013) que el conectivismo describe el aprendizaje como una oportunidad de compartir conocimientos y experiencias con otros individuos.

Los propios Solórzano y García (2016) indican los siguientes seis principios sobre el conectivismo:

1. Base psicopedagógica del aprendizaje en red: se separa de la idea de que el aprendizaje es propio y exclusive del individuo, para ser patrimonio del colectivo.

2. Capacidad para elegir la información a estudiar de forma crítica.

3. Carácter social del aprendizaje, ya que se usan las redes sociales para construir el conocimiento. 
4. Dado que los usuarios de las redes sociales son "prosumidores", es evidente que se da un aprendizaje activo.

5. Se fomenta la integración de los individuos, ya que forman parte de una red.

6. Tal y como se puede entender en base a los puntos anteriores, la tecnología digital tiene un papel crucial en todo el proceso educativo.

Las estadísticas actuales muestran como los estudios de grados universitarios han aumentado en los últimos años un $5 \%$ en España y los estudios de máster un $26 \%$. Además, la formación usando Internet como principal herramienta ha crecido un $900 \%$ desde el año 2000 a nivel mundial, tal y como afirma Muñiz (2017) en El Mundo.

En esta misma línea, tal y como apuntan Viñals y Cuenca (2016), es posible dibujar una sociedad inmersa en la "era digital", donde todos sus ciudadanos están expuestos a un entorno en constante cambio promovido por los avances digitales e Internet, ya que se han creado nuevas formas de comunicación, trabajo e información que solidifican la "sociedad red" (Castells, 2006). No obstante, tal y como apuntan Bringué y Sádaba (2009), manejar los diferentes recursos tecnológicos no es suficiente ya que además hay que tener una adecuada competencia digital. Y es que las TIC han traído consigo nuevos alfabetismos propios del siglo XXI, las cuales añaden competencias que se nutren de la práctica con los diferentes recursos digitales y que los jóvenes desarrollan principalmente en su ocio y tiempo libre (Busquet, Medina \& Ballano, 2013; Viñals \& Cuenca, 2016).

Al hablar de las corrientes pedagógicas actuales donde el alumno es el protagonista de su proceso educativo, es decir, de las corrientes paidocéntricas, Casanova, Pérez, Mar, Chua, Guzmán \& Vincent. (2016), afirman que las ventajas que esta teoría conlleva es que es posible compartir, colaborar, discutir y reflexionar con otros, el aprendizaje deja de ser individualista para pasar a cooperativo y colaborativo; en el primero es el docente el que diseña y mantiene casi por completo el control en la estructura de interacciones y de los resultados que se han de obtener; y en el segundo es lo contrario, los alumnos diseñan como se llevará a cabo la estructura de interacciones y mantiene el control sobre las diferentes decisiones que repercutirán en su aprendizaje; y se propicia espacios en los cuales se da el desarrollo de habilidades individuales y grupales de la discusión entre los estudiantes al momento de explorar nuevos conceptos.

Es, por tanto, que el aprendizaje se diferencia al producido en el pasado en que actualmente puede ser entendido como diverso y desordenado, y el conocimiento en red se sustenta en la creación de contenidos e información.

No obstante, es importante recordar qué es el e-learning y cuáles son las principales diferencias con respecto al aprendizaje presencial, para ello es posible referenciar el trabajo de Cabero y Gisbert (2005), recogido en la siguiente tabla (Tabla 2). 
Tabla 2:

Características de la formación presencial y en red

\begin{tabular}{|c|c|}
\hline Formación basada en la red & Formación presencial tradicional \\
\hline $\begin{array}{l}\text { - Permite que los estudiantes vayan a su propio } \\
\text { ritmo de aprendizaje } \\
\text { - Es una formación basada en el concepto de } \\
\text { formación en el momento en que se necesita } \\
\text { (Just in time training) } \\
\text { - Permite la combinación de diferentes materiales } \\
\text { (auditivos, visuales y audiovisuales) } \\
\text { - Con una sola aplicación puede atenderse a un } \\
\text { mayor número de estudiantes } \\
\text { - El conocimiento es un proceso activo de } \\
\text { construcción } \\
\text { - Tiende a reducir el tiempo de formación de las } \\
\text { personas } \\
\text { - Tiende a ser interactiva, tanto entre los } \\
\text { participantes en el proceso (profesor y } \\
\text { estudiantes) como con los contenidos } \\
\text { - Tiende a realizarse de forma individual, sin que } \\
\text { ello signifique la renuncia a la realización de } \\
\text { propuestas colaborativas } \\
\text { - Puede utilizarse en el lugar de trabajo y en el } \\
\text { tiempo disponible por parte del estudiante } \\
\text { - Es flexible } \\
\text { - Tenemos poca experiencia en su uso } \\
\text { - No siempre disponemos de los recursos } \\
\text { estructurales y organizativos para su puesta en } \\
\text { funcionamiento }\end{array}$ & $\begin{array}{l}\text { - Parte de una base de conocimiento, y el } \\
\text { estudiante debe ajustarse a ella } \\
\text { - Los profesores determinan cuándo y } \\
\text { cómo los estudiantes recibirán los } \\
\text { materiales formativos } \\
\text { - Parte de la base de que el sujeto recibe } \\
\text { pasivamente el conocimiento para } \\
\text { generar actitudes innovadoras, críticas e } \\
\text { investigadoras } \\
\text { - Tiende a apoyarse en materiales } \\
\text { impresos y en el profesor como fuente } \\
\text { de presentación y estructuración de la } \\
\text { información } \\
\text { - Tiende a un modelo lineal de } \\
\text { comunicación } \\
\text { - La comunicación se desarrolla } \\
\text { básicamente entre el profesor y el } \\
\text { estudiante } \\
\text { - La enseñanza se desarrolla de forma } \\
\text { preferentemente grupal } \\
\text { - Puede prepararse para desarrollarse en } \\
\text { un tiempo y en un lugar } \\
\text { - Se desarrolla en un tiempo fijo y en aulas } \\
\text { específicas } \\
\text { - Tiende a la rigidez temporal } \\
\text { - Tenemos mucha experiencia en su } \\
\text { utilización } \\
\text { - Disponemos de muchos recursos } \\
\text { estructurales y organizativos para su } \\
\text { puesta en funcionamiento }\end{array}$ \\
\hline
\end{tabular}

Fuente: tomado de Cabero y Gisbert, (2005).

\section{Conclusiones}

Una vez expuestas todas las referencias y antecedentes relacionados con el Conectivismo, se puede llegar a concluir claramente, tal y como se apoyaba en las tesis iniciales, que esta corriente teórica supone más una evolución del conocimiento teórico existente que una auténtica revolución teórica.

El motivo principal por el que se puede llegar a pensar lo contrario se puede encontrar 
en la analogía que se suele realizar entre la revolución sociotecnológica actual y lo que supone el Conectivismo al ámbito pedagógico. Mientras en el aspecto sociotecnológico no hay duda alguna de que estamos inmersos en una auténtica revolución que modifica las bases establecidas, en el campo de la pedagogía y el conocimiento la evolución supone pequeños cambios de perspectivas, nuevas formas de afrontar los desafíos sociopedagógicos y nuevas posibilidades de enfocar los retos futuros en el ámbito educativo que se aprovechan de los amplios conocimientos actuales para reforzar sus cimientos y afrontar con mayores garantías un futuro demasiado incierto.

En la actualidad, el enfoque teórico educativo del conectivismo nos permite multitud de ventajas que han favorecido su imposición como nuevo paradigma teórico de la educación para una amplia mayoría de nuevos investigadores. Estas ventajas pueden ser resumidas en las siguientes:

- Continuidad respecto al conocimiento educativo existente, ya que los principios conectivistas no suponen una ruptura, sino un reenfoque en la mayoría de circunstancias.

- Facilidad de aplicación de los principios conectivistas a las nuevas tecnologías y metodologías educativas, al estar en consonancia con la mayoría de las tendencias actuales.

- Alta capacidad de adaptación a las nuevas generaciones de alumnos 'Nativos Digitales' que se identifican cada vez más con las necesidades descritas desde el Conectivismo.

- Mayor sintonía entre las distintas ciencias y campos de estudios, al ser el Conectivismo un enfoque que bebe de diversos ámbitos teóricos para desarrollarse (psicología social, tecnologías de la comunicación, neurociencia, informática, etc.).

- Mayor comprensión de la situación educativa actual y los desafíos futuros, ya que el conectivismo contempla una mayor comprensión de una sociedad en permanente cambio y un conocimiento en constante expansión.

No obstante, no se debe olvidar el importante papel que juega el docente en el aprendizaje del aprendiz, ya que su labor supone ejercer de puente activo sobre comportamientos e intereses del aprendiz que no necesariamente están orientados al aprendizaje. El docente debe ejercer de apoyo, de 'traductor', para facilitar la abstracción y el aprendizaje en los contextos educativos.

En definitiva, se puede vislumbrar y justificar una más que posible expansión y popularización del Conectivismo en el ámbito educativo teniendo en cuenta la evolución actual de la tecnología de la información y las redes sociales, que de momento avanzan imparables a expandirse a todos los aspectos de la vida social de nuestra comunidad, y por lo que el ámbito educativo debe adecuarse a las nuevas perspectivas integrando de la mejor forma posible sus principios para utilizarlos con el fin de garantizar a los futuros alumnos la mejor educación posible. 
También es posible afirmar que, tal y como se ha mostrado a lo largo de este trabajo, el conectivismo se presenta como la base metodológica de los procesos de e-learning e incluso se puede llegar a pensar que es una teoría desarrollada principalmente para este tipo de enseñanza a distancia.

\section{Referencias}

Abrio, A. \& Bermúdez, J. (2017).Hacia dónde va el rol del docente en el siglo XXI? Estudio comparativo de casos reales basados en las teorías constructivistas y colectivista. Hekademos: revista educativa digital, (22), 84-92. Recuperado de: https://dialnet.unirioja.es/descarga/articulo/6280736.pdf

Aguilar, J. \& Mosquera, D. (2015). Middleware Reflexivo para la gestión de Aprendizajes Conectivistas en Ecologías de Conocimientos (eco-conectivismo). Latin American Journal of Computing, 2(2), 25-32. Recuperado de: http://lajc.epn.edu.ec/index.php/LAJC/article/view/87

Arenas, A. (2018). La escuela bajo los preceptos de la teoría del caos: Incertidumbre, caos, complejidad, lógica difusa y bioaprendizajes/The School under the Provisions of Chaos Theory: Uncertainty, Chaos, Complexity, Fuzzy logic and Biological learnings. Revista Internacional de Educación y Aprendizaje, 6(1), 1-9. Recuperado de: https://journals.epistemopolis.org/index.php/educacion/article/view/360/1087

Ausubel, D. (1964). Some psychological aspects of the structure of knowledge. In: ELAM, S. (Ed.) Education and the structure of knowledge. Illinois: Rand MacNally.

Barnes, C. \& Tynan, B. (2007). The adventures of Miranda in the brave new world: learning in a Web 2.0 millennium. Research in Learning Technology, 15(3), 189200. Recuperado de: https://journal.alt.ac.uk/index.php/rlt/article/view/954/1205

Bawden, D., Robinson, L., Anderson, T., Bates, J., Rutkauskiene, U. \& Vilar, P. (2008). Towards Curriculum 2.0: library/information education for a Web 2.0 world. Library and information Research, 31(99), 14-25. Recuperado de: http://citeseerx.ist.psu.edu/viewdoc/download?doi=10.1.1.920.5370\&rep=rep1\&ty pe $=p d f$

Bringué, X. \& Sádaba, CH. (2009). La Generación Interactiva en España. Niños y adolescentes ante las pantallas. Pamplona: Ariel. Recuperado de: http://goo.gl/FIXezM.

Brown, T. H. (2006). Beyond constructivism: navigationism in the knowledge era. On the Horizon, 14(3), 108-120. https://doi.org/10.1108/10748120610690681

Bruner, J. (1966). Toward a theory of instruction. Cambridge: Harvard University Press.

Bruner, J. (2001). El proceso mental en el aprendizaje. Barcelona: Narcea. 
Busquet, J., Medina, A. \& Ballano, S. (2013). El uso de las TRIC y el choque cultural en la escuela. Encuentros y desencuentros entre maestros y alumnos. Revista Mediterránea de Comunicación, 4(2), 115-135. DOI: http://dx.doi.org/10.14198/MEDCOM2013.4.2.06

Cabero, J. \& Gisbert, M. (2005). Formación en Internet. Guía para el diseño de materiales didácticos. Sevilla: MAD.

Casanova, M., Pérez, M., Mar, M., Chua, A., Guzmán, J, \& Vincent, M. (2016). Medios Emergentes Digitales: "Una Experiencia en el Aula". Recuperado de: https://www.cidui.org/revistacidui/index.php/cidui/article/view/897

Castells, M. (1999). La era de la información I. La sociedad red. Madrid: Alianza.

Castells, M. (2006). La sociedad red: una visión global. Madrid: Alianza Editorial.

Cathalifaud, A. (2014). La construcción del conocimiento fundamentos epistemológicos del constructivismo. Investigaciones Sociales, 8(12), 271-287. Recuperado de: http://revistasinvestigacion.unmsm.edu.pe/index.php/sociales/article/view/6897/61 04

Colom, A. (2005). Teoría del Caos y Práctica Educativa. Revista Galega do Ensino, 13(47), 1325-1343.

Recuperado

de: http://dialnet.unirioja.es/descarga/articulo/2554834.pdf

Downes, S. (2005). Feature: E-learning 2.0. Elearn magazine, 2005(10), 1-15.

Downes, S. (2008). Connectivism and its critics: What connectivism is not. Recuperado de: http://www.downes.ca/post/53657

Downes, S. (2016). The Theory of Connectivism: Can It Explain and Guide Learning in the Digital Age? Journal of Higher Education Theory and Practice, 8, 11-26. Recuperado de: http://m.www.nabusinesspress.com/JHETP/ForoughiA_Web15_5_.pdf

Duffy, T. M., \& Jonassen, D. (2013). Constructivism and the technology of instruction: A conversation. London: Routledge.

Esteve, F. (2016). Bolonia y las TIC: de la docencia 1.0 al aprendizaje 2.0. La cuestión universitaria, (5), 58-67. Recuperado de 2018 de: http://polired.upm.es/index.php/lacuestionuniversitaria/article/view/3337/3402

Flórez, S., Pérez, J. \& Amaya, Á. (2017). Sinergia entre e-Learning y e-Commerce. Tecnología Investigación y Academia, 5(1), 91-106. Recuperado de: http://revistas.udistrital.edu.co/ojs/index.php/tia/article/view/12014/pdf

Forrest, S. \& Jones, T. (1994). Modeling complex adaptive systems with Echo, in R.J. Stonier and X.H. Yu, eds. Complex Systems: Mechanisms of Adaptation. IOS Press, Amsterdam. pp. 3-21. 
Forster, T. (2008). Connectivism Is. Online Connectivism Conference: University of Manitoba. Recuperado de: https://archive.org/details/ERIC_EJ815759/page/n0

Freud, S. (1984). La interpretación de los sueños: obras completas. Buenos Aires: Amorrortu, 4.

Fuentes, F. (2017). El cambio de conceptos y teorías en el conocimiento científico y ordinario. Educación y Humanismo, 19(33), 253-270. DOI: http://dx.doi.org/10.17081/eduhum.19.33.2643

Gagné, R. (1971). Las condiciones del aprendizaje. Madrid: Aguilar.

Ganino, G. (2018). Didáctica de la web conference en el ámbito universitario. Educación y Humanismo, 20(34), 15-35. DOI: http://dx.doi.org/10.17081/eduhum.20.34.2856

Harasim, L. (1995). Learning networks: A field guide to teaching and learning online. MIT press.

Hardman, E. (2015). How pedagogy 2.0 can foster teacher preparation and community building in special education. Social Inclusion, 3(6), 42-55. Recuperado de: https://www.cogitatiopress.com/socialinclusion/article/view/415/415

Holland, J. H. (1996). Hidden order: how adaptation builds complexity. New York, NY: Addison-Wesley.

Hug, T. (2007). Didactics of microlearning. New York, NY: Waxmann Verlag.

Imran, A. S., \& Kowalski, S. J. (2014). HIP-A technology-rich and interactive multimedia pedagogical platform. In International Conference on Learning and Collaboration Technologies, (14), 151-160. Springer, Cham. Recuperado de: https://link.springer.com/chapter/10.1007\%2F978-3-319-07482-5_15

Islas, C. \& Delgadillo, O. (2016). La inclusión de TIC por estudiantes universitarios: una mirada desde el conectivismo. Apertura: Revista de innovación educativa, 8(2), 116-129. Recuperado de: https://dialnet.unirioja.es/descarga/articulo/5658842.pdf

Konig, D. (1936). Theorie der endlichen und unendlichen Graphen, Leipzig: Akad. Verlagsges. $\mathrm{mbH}$.

Latour, B (1999). "On Recalling ANT," in Law and Hassard, eds., ActorNetwork Theory and After. Oxford: Blackwell Publishers, pp. 15-26.

Laurillard, D. (1993). Rethinking University Teach-ing: A Framework for the Effective Use of Educational Technology. London: Routledge.

Laurillard, D. (1999). A Conversational Framework for Individual Learning Applied to the Learning. Systems Research and Behavioural Science. (16), 113-122. http://dx.doi.org/10.1002/(SICI)1099-1743(199903/04)16:2\%3C113::AIDSRES279\%3E3.0.CO;2-C 
Laurillard, D. (2002) Rethinking university teaching: A conversational framework for the effective use of learning technologies (2nd ed.). London: Routledge Falmer

Law, J. (1999). "After ANT: Complexity, Naming and Topology." In Hassard, J \& Law, J. (ed.) Actor-Network Theory and After. Oxford: Blackwell Publishers.

Levin, S.A. (2002). Complex adaptive systems: Exploring the known, the unknown and the unknowable. Bulletin (new series) of the American Mathematical Society, 40(1), 319.

Lewin, K. (1938). The conceptual representation and measurement of psychological forces. Duke University. Contributions to Psychological Theory, 1(4), 247-255.

Lima, G. (2017). Dos modelos: aprendizaje colaborativo on line y comunidad de investigación. In IV Jornadas de TIC e Innovación en el Aula (La Plata, 2017). Recuperado de: http://sedici.unlp.edu.ar/handle/10915/65783

Lozares, C. (2005). Bases sociometodológicas para el análisis de redes sociales, ARS. Empiria: Revista de Metodología de Ciencias Sociales. 10(2), 9-35. Recuperado de: http://e-spacio.uned.es/fez/eserv.php?pid=bibliuned:Empiria-2005-CBBC327EF8AE-F864-2A0C-B2AC936A8A8C\&dsID=Documento.pdf

Maldonado, C. (2017). Educación compleja: Indisciplinar la sociedad. Revista Educación y Humanismo, 19(33), 234-252. DOI: https://doi.org/10.17081/eduhum.19.33.2642

Masie, E. (2006). Nano-Learning: miniaturization of design. Chief Learning Officer, 5(1), 17-25.

Mayer, R. (1992). Cognition and instruction: Their historic meeting within educational psychology. Journal of Educational Psychology, (84), 405-412.

Martín, T., García, J., \& Ramirez, R. (2004). Aplicaciones de la Teoría de la Conversación a entornos docentes telemáticos. T. Yousef. Recuperado de: https://www.researchgate.net/publication/237615442_Aplicaciones_de_la_Teoria_ de_la_Conversacion_a_entornos_docentes_telematicos

Mcloughlin, C. \& Lee, M. (2007). Social software and participatory learning: Pedagogical choices with technology affordances in the Web 2.0 era. In ICT: Providing choices for learners and learning. Proceedings ascilite Singapore 2007. Recuperado de: http://citeseerx.ist.psu.edu/viewdoc/download?doi=10.1.1.471.2008\&rep=rep1\&ty pe $=p d f$

McLoughlin, C. \& Lee, M. J. (2008). The 3 P's of pedagogy for the networked society: Personalization, participation, and productivity. International Journal of Teaching and Learning in Higher Education, 20(1). 10-27. Recuperado de: http://www.isetl.org/ijtlhe/articleView.cfm?id=395

Moreno, J. (1962). Fundamentos de la Sociometría. Buenos Aires: Piados.

Mukamel, R., Ekstrom, A., Kaplan, J., Iacoboni, M. \& Fried, I. (2010). Single-neuron 
responses in humans during execution and observation of actions. Current Biology. 20(8), 750-6. https://doi.org/10.1016/j.cub.2010.02.045

Muñiz, M. (2017, 26 de septiembre). El 'e-learning', un modelo de formación que gana cada vez más adeptos. El Mundo. Recuperado de: https://www.elmundo.es/extras/formaciononline/2017/09/26/59ca90a1ca4741900e8b4601.html

Nobles, D., Londoño, L., Martínez, S., Ramos, A., Santa, G. \& Cotes, A. (2016). Tecnologías de la comunicación y relaciones interpersonales en jóvenes universitarios. Educación y Humanismo, 18(30), 14-27. DOI: http://dx.doi.org/10.17081/eduhum.18.30.1311

Organista, J., Mcanally, L. \& Lavigne, G. (2013). El teléfono inteligente (smartphone) como herramienta pedagógica. Apertura: Revista de innovación Educativa, 5(1), 6-19. Recuperado

de: http://www.udgvirtual.udg.mx/apertura/index.php/apertura/article/view/372/311

Osuna, J. B. \& Almenara, J. (2015). Replanteando el e-learning: hacia el e-learning 2.0. Campus virtuales, 2(2), 76-87. Recuperado de: http://www.uajournals.com/ojs/index.php/campusvirtuales/article/view/40

Papert, S. (1995): La máquina de los niños. Replantearse la educación en la era de los ordenadores. Barcelona: ed. Paidós.

Pask, G. (1975). "Conversation, Cognition and Learning". Amsterdam: North Holland Publishing Co.

Piaget, J. (1954). The Construction Of Reality In The Child. London: Routledge.

Polsani, P. R. (2003). Network learning. In K. Nyíri (Ed.), Mobile learning: Essays on philosophy, psychology and education (pp. 139-150). Vienna: Pasagen Verlag.

Rizzolatti, G., Fadiga, L., Matelli, M., Bettinardi, V., Paulesu, E., Perani, D. \& Fazio, F. (1996) Localization of grasp representation in humans by PET: 1 . Observation versus execution. Experimental Brain Research. (111), 246-252.

Rivera, P., Alonso, C. \& Sancho, (2017). Desde la educación a distancia al e-Learning: emergencia, evolución y consolidación. Educación y Tecnología, (10), 1-13. Recuperado de: https://dialnet.unirioja.es/descarga/articulo/6148504.pdf

Rock, I. \& Palmer, S. (1990). The legacy of Gestalt psychology. Scientific American, 263(6), 84-91.

Rodríguez, J. (2016). La renovación pedagógica: del instruccionismo intelectualista al holismo educativo. Tendencias Pedagógicas, (27), 43-76. Recuperado de: https://revistas.uam.es/tendenciaspedagogicas/article/view/2999

Salinas, J. \& Marín, V. (2015). Pasado, presente y futuro del microlearning como estrategia para el desarrollo profesional. Campus Virtuales, 3(2), 46-61. Recuperado de: 
http://uajournals.com/ojs/index.php/campusvirtuales/article/view/59/58

Sangrà, A., \& Wheeler, S. (2013). Nuevas formas de aprendizaje informales:¿ O estamos formalizando lo informal?. RUSC. Universities and Knowledge Society Journal, 10(1), 15-29.DOI: http://dx.doi.org/10.7238/rusc.v10i1.1689\%3E

Santamaría, F. (2010). La era conectiva: por el desorden natural de los artefactos y nodos. Recuperado de: http://www.nodosele.com/editorial/wpcontent/uploads/2010/01/Introduccion.FernandoSantamaria.Conociendoelconocimi ento2.pdf

Schutt, F. (1995). El pensamiento freudiano en 1895: Estudios sobre la histeria cien años después. Anuario de psicología/The UB Journal of psychology, (67), 9-14.

Siemens, G. (2004). Connectivism: A learning theory for the digital age. International journal of instructional technology and distance learning, 2(1), 3-10.

Siemens, G. (2006). Knowing knowledge. Morrisville, Estados Unidos: Lulu.com.

Siemens, G., \& Conole, G. (2011). Special issue-Connectivism: Design and delivery of social networked learning. International Review Of Research in Open and Distance Learning, 12(3). Recuperado de: https://www.researchgate.net/publication/50888824_Special_Issue__Connectivism_Design_and_Delivery_of_Social_Networked_Learning

Sobrino, Á. (2014). Aportaciones del conectivismo como modelo pedagógico postconstructivista. Propuesta educativa, (42), 39-48. Recuperado de: http://www.scielo.org.ar/scielo.php?script=sci_arttext\&pid=S199577852014000200005

Solórzano, F. \& García, A. (2016). Fundamentos del aprendizaje en red desde el conectivismo y la teoría de la actividad. Revista Cubana de Educación Superior, 35(3), 98-112. Recuperado de: http://scielo.sld.cu/scielo.php?script=sci_arttext\&pid=S025743142016000300008\&lng=es\&tIng=pt

Vidal, J. P. (2016). Teoria de sistemas autopoiéticos y la actor network theory: ¿Hacia una convergencia posestructuralista? INTERthesis: Revista Internacional Interdisciplinar, 13(2), 38-57. Recuperado de: https://dialnet.unirioja.es/descarga/articulo/5562180.pdf

Viñals B. \& Cuenca, J. (2016). El rol del docente en la era digital. Revista Interuniversitaria de Formación del Profesorado, 30(2), 103-114. Recuperado de: http://www.redalyc.org/pdf/274/27447325008.pdf

Vygotsky, L.S. (1932). Mind in society: The development of higher psychological processes (1932). Cambridge, MA: Harvard University Press.

Wertheimer, M. \& Riezler, K. (1944). Gestalt theory. Social Research, (5), 78-99. 
Wertsch, J. (1988). Vygotsky y la formación social de la mente. Barcelona, España: Paidós.

Zapata-Ros, M. (2015). Teorías y modelos sobre el aprendizaje en entornos conectados y ubicuos. Bases para un nuevo modelo teórico a partir de una visión crítica del "conectivismo". Education in the Knowledge Society, 16(1), 69-102. Recuperado de:

http://campus.usal.es/ revistas_trabajo/index.php/revistatesi/article/view/eks2015 16169102 